Original

\title{
Procedimiento Cabestrillo Autólogo versus Sintético: Tasa de éxito y frecuencia de obstrucción del tracto urinario inferior
}

\author{
Ricardo A. Natalin, Cassio Riccetto, Renato Nardi Pedro, Alessandro Prudente, \\ Guillermo Salvador Navarrete, Paulo C. Rodrigues Palma
}

Departamento de Urología. Universidad Estatal de Campinas - UNICAMP - Brasil.

\begin{abstract}
Resumen
Introducción: Disfunción en el vaciamiento y retención urinaria son complicaciones frecuentes del cabestrillo. Recientemente se han incluido en el arsenal para el soporte sub-uretral, suministros sintéticos tales como la malla de polipropilenol.

Métodos: Se llevó a cabo un Estudio prospectivo no aleatorizado. El procedimiento cabestrillo se realizó en 436 pacientes consecutivos con IUE (Incontinencia Urinaria de Esfuerzo). Las pacientes fueron divididas en grupos según el tipo de malla, en autólogos 210 y 226 sintéticos. Las pacientes fueron evaluadas en cuanto a condición de vaciamiento, continencia y satisfacción. Los resultados quirúrgicos fueron evaluados en función de la clasificación de Blaivas. Se consideró significativo p<0,05. Resultados: La media de edad fue 48,4 años. El tiempo medio operatorio para los Cabestrillo autólogos fue de 107,6 minutos frente a 20,6 min para el sintético. El promedio de hospitalización fue de 36 horas para autólogos frente a 24 horas para los sintéticos y la media de seguimiento fue de 25 meses. Se encontró $87,1 \%$ y 92,9\% de las pacientes continentes en los grupos autólogos y sintéticos, respectivamente. Fallo en el procedimiento se presentó en un 7\% en el grupo autólogo y 4,8\% en el sintético (p=0,1961). La tasa global de obstrucción del tracto urinario inferior fue de 4,5\% (20/436), cuando se analizó por grupos, encontramos en el grupo autólogo una tasa de 8,5\% (18/210), en comparación con el 0,9\% en el grupo sintético $(2 / 226)(\mathrm{p}=0,000126)$.

Conclusiones: Cabestrillos autólogos y sintéticos presentan las tasas de éxito comparables, sin embargo la obstrucción del tracto urinario inferior fue más común entre las pacientes tratadas con fascias autólogas.
\end{abstract}

Palabras claves: Cabestrillo. Sling, autólogo Sintético. Malla. Incontinencia urinaria.

\section{Autologous versus Synthetic sling procedure: Success rate and bladder outlet obstruction rates \\ Abstract}

Objectives: Voiding dysfunction and urinary retention were frequent complications of sling. More recently synthetic supplies such as polypropylene mesh have been included in the armamentarium for sub urethral support.

Methods: A prospective non randomized study was conducted. Sling procedure was performed in 436 consecutive patients with SUI. Patients were divided in groups according to mesh type, being 210 on autologous and 226 on synthetic. Patients were surveyed on voiding condition, continence and satisfaction. Surgical results were assessed according to Blaivas classification. Results were considered significant $\mathrm{p}<0.05$.

Results: Mean patient age was 48,4years. Mean operative time for autologous sling was of 107,6 min versus 20,6min for synthetic. Mean hospitalization was $36 \mathrm{~h}$ on autologous versus $24 \mathrm{~h}$ on synthetic and mean follow up was 25 months. We found $87,1 \%$ and $92,9 \%$ of patients continent in autologous and synthetic group, respectively. Failure was presented by $7 \%$ in autologous and $4,8 \%$ in synthetic group ( $\mathrm{p}=0.1961)$. The global rate of BOO was of $4,5 \%(20 / 436)$, when analysed by groups we found on autologous a rate of $8.5 \%(18 / 210)$, compared to $0.9 \%$ on synthetics $(2 / 226)(\mathrm{p}=0.000126)$.

Conclusions: Autologous and synthetic slings presented comparable success rates, however BOO was more common among patients treated by autologous slings.

Keywords: Sling. Autologous. Synthetic. Mesh. Urinary incontinence.

$\mathrm{L}$ a disfunción en el vaciado y la retención urinaria son complicaciones frecuentes de la uretropexia y el Cabestrillo uretral. Sobre la base de la Teoría Integral, Petros y Ulmsten propusieron el procedimiento de la cinta vaginal sin tensión para el tratamiento de mujeres con IUE ${ }^{1}$. Desde los primeros informes de procedimientos de Cabestrillo vaginal sintético, éste se ha convertido en el estándar de oro en el tratamiento de la incontinencia urinaria de esfuerzo (IUE) ${ }^{2}$. El uso de la fascia autóloga implica un procedimiento más invasivo que clínicamente se traduce en un mayor tiempo operatorio, con aumento de la morbilidad y por lo tanto, complicaciones, tales como dolor, sangrado y la formación de cicatriz ${ }^{3}$. 
Los injertos sintéticos, como la malla de polipropileno, pueden superar la necesidad de tejido autólogo, disminuyendo el tiempo operatorio y la recuperación postoperatoria. Los procedimientos para corregir la IUE están diseñados para restablecer el soporte de la unión uretrovesical y, en casos de disfunción esfinteriana intrínseca, mejorar la coaptación de la uretra. Guatelli et al, reportaron el 8,5\% de tasa de obstrucción posterior al procedimiento de cabestrillo autólogo ${ }^{4}$, mientras que la tasa de obstrucción con las mallas de polipropileno fue del $3,7 \% 5$.

El objetivo de este estudio fue comparar las tasas de éxito y de obstrucción del tracto urinario inferior, posterior al procedimiento Cabestrillo para la IUE, con dos diferentes materiales, sintético y autólogo.

\section{PACIENTES Y MÉTODOS}

Entre julio y diciembre de 2005 se realizó un estudio prospectivo no aleatorizado en el Centro Médico de la Universidad de Campinas. El protocolo de estudio fue aprobado por la Junta de Revisión Institucional y se obtuvo de cada paciente el consentimiento informado. Los criterios de exclusión comprendían mujeres con antecedentes de enfermedades neurológicas, derivación urinaria, divertículos vesicales, fístula uretrovaginal o vesicovaginal, antecedente de radioterapia o cirugía pélvica. Además, las pacientes que presentaron contracciones involuntarias del detrusor durante el llenado vesical o flujo máximo (Qmax) inferior a $15 \mathrm{ml} / \mathrm{seg}$ y/o con el residuo de orina postmicción de más del 20\% del volumen, fueron excluidas del estudio, pero las que tenían sintomas irritativos sin demostrar urodinámicamente contracciones involuntarias se incluyeron, aunque se demostró urodinámicamente que la inestabilidad del detrusor no tenía un efecto significativo en el resultado quirúrgico. Esta decisión se basó en el concepto referente a la mejoria postoperatoria de la urgencia sensorial.

Un total de 436 pacientes con IUE, todas confirmadas urodinámicamente, se sometieron al procedimientos de cabestrillo. La prueba de esfuerzo fue positiva en todas las pacientes que participaron en el estudio. En la mayoría de las pacientes había fracasado al menos un procedimiento anti-incontinencia (media del 62,5\%). El procedimiento más común fue la Plicatura de Kelly en el $73 \%$ de los casos. Las pacientes fueron divididas en dos grupos según el tipo de malla utilizada para el soporte de la uretra anterior. Ambos grupos fueron similares en las características demográficas, antropométricas, clínicas y de estudio urodinámico. El Cabestrillo Autólogo pubo- vaginal (con fascia de recto abdominal) se realizó en 210 pacientes y el Cabestrillo con malla sintética se realizó en 226 pacientes (con la cinta vaginal sin tensión-TVT).

El Cabestrillo autólogo se realizó a través de una vía combinada abdomino-vaginal y el tejido se obtuvo de la fascia de recto abdominal. El Cabestrillo Sintético se realizó según lo descrito por Ulmsten et $\mathrm{al}^{6}$ bajo anestesia general o espinal. La TVT difiere del procedimiento cabestrillo pubovaginal autólogo en la manera en que el material del Cabestrillo suburetral se colocó en la uretra media en lugar de el cuello vesical.

Las pacientes fueron seguidas periódicamente en la clínica de Urología de nuestra institución a la $1^{\mathrm{a}}$ semana post-quirúrgica, 30 días, 90 días, 180 días y luego cada año. Ellas fueron encuestadas en cuanto a su vaciamiento, la continencia y la satisfacción subjetiva. Los resultados quirúrgicos fueron evaluados de acuerdo a la clasificación de Blaivas et al. ${ }^{7}$. Las pacientes con síntomas miccionales después de la cirugía fueron investigadas y tratadas de acuerdo a los hallazgos urodinámicos. Los resultados se analizaron utilizando la prueba $\mathrm{T}$ pareada para datos pareados y la prueba $\mathrm{T}$ de Student y la prueba de chi-cuadrado para datos independientes, $\mathrm{P}<0,05$ considerado estadísticamente significativo.

\section{RESULTADOS}

La edad media de las pacientes fue 48,4 años (3468) con una mediana de paridad de 3,4 (3-12). La media del tiempo operatorio para el Cabestrillo autólogo fue de 107,6 minutos y para el Cabestrillo sintético de 20,6 min (Fig. 1). La media del período de hospitalización fue de $36 \mathrm{~h}$ (24h-48h) para el grupo del Cabestrillo autólogo en comparación con una media de 24 h (12h-36h) para el grupo del Cabestrillo sintético. La media del tiempo de seguimiento postoperatorio fue de 25 meses (12-48 meses).

Según los criterios de Blaivas et al, 183/210 pacientes $(87,1 \%)$ y $210 / 226(92,9 \%)$ fueron continentes en el grupo autólogo y grupo sintético, respectivamente. Fallos se presentaron en el 7\% en el grupo autólogo y $4,8 \%$ en el grupo sintético ( $\mathrm{p}=0,1961)$. El cuestionario ICQ-SF mostró una mejoría significativa en todas las cuestiones en comparación con la evaluación preoperatoria (93\% de las pacientes informaron mejoría en 


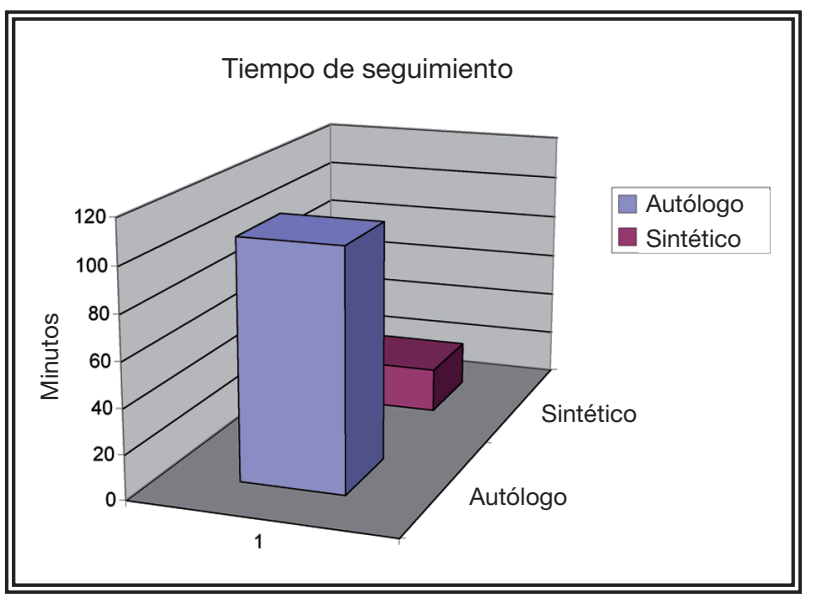

FIGURA 1. Gráfico que muestra el tiempo operatorio entre los grupos.

los sintomas urinarios después de la cirugía, en ambos grupos). Durante el período de seguimiento, todas las pacientes presentaron una prueba de esfuerzo negativa.

Entre las pacientes con incontinencia el uso de compresas sanitarias fue inferior a uno por día. La mediana del tiempo entre el procedimiento antiincontinencia y el diagnóstico de Obstrucción del Tracto Urinario Inferior fue de nueve meses (3-96 meses). Todas las pacientes obstruidas sufrieron del síndrome refractario urgencia/frecuencia, con volumen residual postmicción variable. El diagnóstico de Obstrucción del Tracto Urinario Inferior, se realizó por medio de historia clínica, diario miccional, examen físico y urodinámica.

La tasa global de Obstrucción del Tracto Urinario Inferior (Fig. 2), evaluada por los hallazgos urodiná-

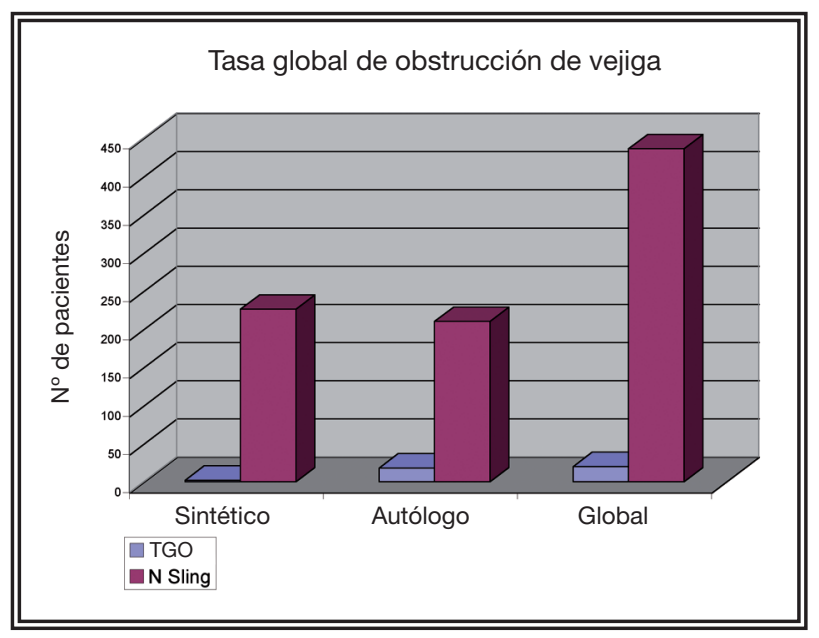

FIGURA 2. Gráfico que muestra la obstrucción del tracto urinario inferior entre los grupos. micos de bajo flujo con alta presión del detrusor, fue de 4,5\% (20/436) de todas los pacientes tratadas con cabestrillo. Cuando se estudió el tipo de Cabestrillo, encontramos en las pacientes que se sometieron al Cabestrillo autólogo una tasa de 8,5\% (18/210), en comparación con el 0,9\% para las que se sometieron al Cabestrillo sintético (2/226) $(\mathrm{p}=0,000126)$.

En los datos urodinámicos se demostró una media de flujo de vaciado de 9,9 ml/s, (3,9-10,2 $\mathrm{ml} / \mathrm{s}$ ) y una media de la presión del detrusor en el flujo máximo de $48 \mathrm{cmH}_{2} \mathrm{O}$, (36-84 $\left.\mathrm{cmH}_{2} \mathrm{O}\right)$. Tres pacientes $(15 \%)$ presentaron un volumen residual post micción superior a $200 \mathrm{ml}$ y siete (35\%) presentaron contracciones del detrusor no inhibidas.

\section{DISCUSIÓN}

El Cabestrillo sin tensión es considerado el Standard de Oro en el manejo de la Incontinencia urinaria de esfuerzo, en el seguimiento a largo plazo $^{8}$; sin embargo, requiere un considerable período de entrenamiento quirúrgico y mucho tiempo para la obtención de la fascia, además del riesgo de obstrucción infravesical y disfunción vesical ${ }^{9}$.

El procedimiento de cabestrillo vaginal presenta un efecto duradero sobre la continencia urinaria sin comprometer la resistencia uretral, con baja morbilidad quirúrgica y alta tasa de satisfacción $(89 \%)^{10}$. Nuestro estudio, confirma la elevada tasa global de éxito de los procedimientos, independientemente del material utilizado.

Los resultados mostraron una tasa de éxito de $87,1 \%$ (183/210 pacientes) y 92,9\% (210/226) para material autólogo y sintético, respectivamente. Nuestros datos están en conformidad con la literatura, como lo demuestra Wadie et al con 92,9\% de tasa de éxito con cabestrillo de polipropileno y $92 \%$ con fascias autólogas ${ }^{3}$. Este resultado es apoyado por el resultado del cuestionario ICQ-SF, mostrando mejoría en comparación con la evaluación preoperatoria, con 93\% de las pacientes reportando mejoría de los síntomas urinarios después de la cirugía.

A pesar de las actuales tasas de éxito, los procedimientos de Cabestrillo se han asociado a importantes tasas de infección del tracto urinario, de incontinencia de urgencia, y de disfunción miccional, a veces incluso requiriendo una segunda cirugía para mejorar el vaciamiento ${ }^{11}$. El aumento de la morbilidad del procedimiento de Cabestrillo, explica en algo la oposición en el pasado para utilizar este 
procedimiento como principal tratamiento quirúrgico de la incontinencia de esfuerzo ${ }^{12}$. Debido al desarrollo de nuevas técnicas quirúrgicas y la ampliación del conocimiento sobre los materiales biomédicos, el procedimiento de Cabestrillo se ha convertido en una atractiva y muy bien tolerada modalidad para el tratamiento de la Incontinencia Urinaria de Esfuerzo. Los materiales Autólogos tienen la ventaja de ser incorporados al tejido vaginal, con un mínimo costo específico, y también evitan que el paciente adquiera una enfermedad infecciosa cuando se comparan con fascia de cadáver (aloinjerto) ${ }^{13}$.

Por otra parte, el cabestrillo autólogo se relaciona con la estancia hospitalaria más prolongada (lo que podría implicar un mayor coste global para el procedimiento) y mayor período de recuperación. Los Materiales sintéticos tiene la ventaja de ser biológicamente inertes, de presentar una mayor resistencia frente a materiales biológicos, de permitir un mínimo de disección y evitan que la paciente sea sometida a una incisión abdominal ${ }^{3}$. En consecuencia, las pacientes permanecen hospitalizadas por un menor período de tiempo y regresan pronto a sus actividades regulares.

Sin embargo los "Kits" quirúrgicos de mallas sintéticas pueden ser más caros, y las mallas pueden presentar erosión y infección ${ }^{14}$. En el presente estudio, el grupo de Cabestrillo sintético presentó un menor tiempo quirúrgico y estancia hospitalaria más breve. La obstrucción del Tracto Urinario Inferior fue la principal complicación tras los procedimientos para la incontinencia. Una cantidad significativa de pacientes se quejaron de disfunción miccional, disuria y dolor abdominal, y estos hallazgos se reproducen de informes en la literatura, donde 4 al 78\% de los pacientes presentó uno o más síntomas miccionales después de procedimientos similares $^{15-17}$.

La obstrucción puede ser presentada no sólo por la disfunción miccional, sino también por las infecciones del tracto urinario o síntomas de vejiga hiperactiva $^{18}$. La obstrucción del Tracto Urinario Inferior parece ser mayor con el Cabestrillo autólogo ${ }^{11}$. Guatelli et al informaron de una tasa de 8,5\% de obstrucción posterior al procedimiento de cabestrillo autólogo ${ }^{4}$ mientras que la tasa entre los cabestrillo con mallas de polipropileno fue de $3,7 \% \%^{5}$. También un estudio del sistema de tratamiento para la incontinencia urinaria, confirmó el hallazgo de alta tasa de Obstrucción del Tracto Urinario Inferior con Cabestrillo autólogo. En este estudio se mostró un 5,8\% de síntomas miccionales o retención urinaria en un grupo de 326 pacientes sometidos a procedimientos de cabestrillo autólogo ${ }^{11}$. De acuerdo con la literatura, nuestro estudio mostró una tasa global de Obstrucción del Tracto Urinario Inferior de $4,5 \%(20 / 436)$ entre los pacientes tratados con Cabestrillo.

En el análisis de los grupos por separado hubo un $8,5 \%$ en tasa Obstrucción del Tracto Urinario Inferior en el grupo de Cabestrillo autólogo, en comparación con el 0,9\% con el cabestrillo sintético (2/226). Una tendencia a la obstrucción en parámetros urodinámicos se demostró con un bajo flujo urinario y la alta presión del detrusor durante la micción.

El costo ha sido una cuestión al considerar un procedimiento quirúrgico e incluye material, gastos de hospital, medicamentos y recuperación postoperatoria. Cuando hay reducción del tiempo quirúrgico hay una reducción en el gasto de la estancia hospitalaria, así como con la anestesia. Al reducir la manipulación del procedimiento hay una recuperación post quirúrgica más rapida y una reducción del tiempo total hospitalario, logrando al final un significativo ahorro de costes relacionados con el procedimiento. Kondo et al. realizaron un estudio comparativo con respecto a TVT y Cabestrillo autólogo, encontrado una diferencia significativa en relación con los costos de ambos procedimientos ${ }^{19}$.

\section{CONCLUSIÓN}

Los Cabestrillo Autólogos y Sintéticos presentan tasas de éxito comparables en el tratamiento de Incontinencia Urinaria de Esfuerzo; sin embargo, la Obstrucción del Tracto Urinario Inferior fué más común entre las pacientes que se sometieron al procedimiento de Cabestrillo autólogo.

\section{REFERENCIAS}

1. Petros P, Ulmsten UI. An integral theory and its method for the diagnosis and management of female urinary incontinence. Scand J Urol Nephrol. 1993;153:1-93.

2. Ulmsten UI, Petros P. Intravaginal slingplasty (IVS): an ambulatory surgical procedure for treatment of female urinary incontinence. Scand J Urol Nephrol. 1995;29(1):75-82.

3. Wadie BS, Edwan A, Nabeeh AM. Autologous fascial sling vs polypropylene tape at short term follow-up: a prospective randomized study. J Urol. 2005;174(3):990-993.

4. Guatelli S, Dall'Oglio B, Schiavon L, Luciano M, Parma P, Galletta V, et al. Pubo-vaginal sling. Arch Ital Urol Androl. 2004; 76(1):46-48. 
5. Kuo HC. Long-term surgical results of pubovaginal sling procedure using polypropylene mesh in the treatment of stress urinary incontinence. Urol Int. 2005;74(2):147-152.

6. Ulmsten U, Henriksson L, Johnson P, Varhos G. An ambulatory surgical procedure under local anesthesia for treatment of female urinary incontinence. Int Urogynecol J Pelvic Floor Dysfunct. 1996;7(2):81-85.

7. Chaikin DC, Rosenthal J, Blaivas JG. Pubovaginal sling for all types of stress urinary incontinence: long term analysis. J Urol 1998;160(4):1312-1316.

8. Boustead GB. The tension-free vaginal tape for treating female stress urinary incontinence. BJU Int. 2002;89(7):687-693.

9. Klutke C, Siegel S, Carlin B, Paszkiewicz E, Kirkemo A, Klutke J. Urinary retention after tension-free vaginal tape procedure: incidence and treatment. Urology. 2001;58(5):697-701.

10. Kuo HC. The surgical results of the pubovaginal sling procedure using polypropylene mesh for stress urinary incontinence. BJU International. 2001;88(9):884-888.

11. Albo ME, Richter HE, Brubaker L, Norton P, Kraus SR, Zimmern $\mathrm{PE}$, et al. Burch Colposuspension versus Fascial Sling to Reduce Urinary Stress Incontinence. N Engl J Med. 2007;356 (21):21432155

12. Ostergard DR. Primary slings for everyone with genuine stress incontinence? The argument against. Int Urogynecol J Pelvic Floor Dysfunct 1997; 8:321-322.

13. Wilson TS, Lemack GE, Zimmern PE. Management of intrinsic sphincteric deficiency in women. J Urol. 2003;169(5) 1662 1669.

14. Clemens JQ, DeLancey JO, Faerber GJ, Westney OL, Mcguire EJ. Urinary tract erosions after synthetic pubovaginal slings: diagnosis and management strategy. Urology. 2000;56(4):589-594.
15. Nilsson CG, Kuuva N. The tension-free vaginal tape procedure is successful in the majority of women with indications for surgical treatment of urinary stress incontinence. $\mathrm{Br} \mathrm{J}$ Obstet Gynaecol. 2001;108(4):414-419.

16. Debodinance P, Delporte P, Engrand JB, Boulogne M. Tensionfree vaginal tape (TVT) in the treatment of urinary stress incontinence: 3 years experience involving 256 operations. Eur $\mathrm{J}$ Obstet Gynecol Reprod Biol. 2002;105(1):49-58.

17. Abouassaly R, Steinberg JR, Lemieux M, Marois C, Gilchrist LI, Bourque JL, et al. Complications of tension-free vaginal tape surgery: a multi-institutional review. BJU Int. 2004;94(1):110113.

18. Deffieux X, Bonnet K, Chevalier N, Gervaise A, Frydman R, Fernández $\mathrm{H}$. Complications urinaires des soutènements sousurétraux. J Gynecol Obstet Biol Reprod. 2005;34(8):745-756.

19. Kondo A, Isobe Y, Kimura K, Kamihira O, Matsuura O, Gotoh $\mathrm{M}$, et al. Urinary complications in sub-urethral sling procedures. J Obstet Gynaecol Res. 2006;32(6):539-544.

Correspondencia autor: Dr. Paulo C. Rodrigues Palma Departamento de Urología

Cidade Universitária "Zeferino Vaz"

Rua: Tessália Vieira de Camargo, 126 CEP: 13083-887

Campinas - SP - Brasil.

Tel: +55 1935217486 .

E-mail autor: ppalma@uol.com.br.

Información artículo: Original - Incontinencia urinaria

Trabajo recibido: julio 2008

Trabajo aceptado: agosto 2008 